\title{
Praxeología y material pedagógico: cátedras de paz.
}

\author{
García Córdoba, Jhuliet Alejandra \\ Corporación Universitaria Minuto de Dios, Zipaquirá, Colombia \\ jgarciaare1@uniminuto.edu.co \\ Pestana Medina, Stephany \\ Corporación Universitaria Minuto de Dios, Zipaquirá, Colombia \\ spestanamed@uniminuto.edu.co \\ González Bohórquez, Myriam Yohana \\ Corporación Universitaria Minuto de Dios, Zipaquirá, Colombia \\ mgonzalez70@uniminuto.edu.co
}

\section{Resumen}

La presente investigación tiene como fin dar a conocer los resultados obtenidos desde el empoderamiento del modelo praxeológico de la Corporación Universitaria Minuto de Dios Centro Regional Zipaquirá en relación a las sesiones de cátedras de paz como herramienta pedagógica de innovación y transformación social. Para lo cual, inicialmente es necesario hacer hincapié en la formación e incidencia que tienen los valores en el aula y en los diferentes procesos educativos. De tal manera, que sea posible identificar la apropiación de los mismos, así como también la significación que se da a partir de la inclusión, teniendo en cuenta, que cada estudiante del semillero Adherencia Cognitiva y Cambio Social del Programa de Trabajo Social potencializa sus habilidades de acuerdo a la inteligencia múltiple que más desarrollada tenga. Lo anteriormente mencionado, con el fin de llegar a un cambio desde modelos educativos como el modelo praxeológico, caracterizado por promover el desarrollo de todas las dimensiones de la persona, partiendo de las potencialidades de cada uno y que tiene en cuenta el conjunto de ideas, creencias, valores, actos, palabras y contextos del estudiante o comunidad (Juliao, 2012), apoyado en valores y competencias ciudadanas; para establecer cátedras de paz desde los estudiantes del Semillero y de esta manera, promover una apropiación del modelo praxeológico hacia el desarrollo de aptitudes de reconocimiento y pertenencia de su entorno; así como también de la realidad de la que forman parte y que requiere de carácter urgente un trabajo conjunto hacia la construcción de paz.

\section{Abstract}

This research aims to report results in the "Peace courses" as pedagogical tools for social innovation and transformation, led by Corporación Universitaria Minuto de Dios at Zipaquira, Colombia, following the Praxeology model. It is important to highlight the influence of moral values in the classroom and in different educational processes, and identify their internalization, as well as the relevance of social inclusion, taking into account that every student in this research group (Adherencia Cognitiva y Cambio Social del Programa de Trabajo Social), strengthen their most developed intelligence type. The objective is to foster change from educational models such as the praxeology model, which aims to promote the development of all personal dimensions, having in mind individual and community skills, ideas, beliefs, values, actions, words, and contexts (Juliao, 2012). All of this supported in citizenship skills, to then develop Peace courses led by participating students in the research group, whose role is to promote an internalization of praxeology to improve a sense of belonging and awareness of participants' own reality to encourage peacebuilding.

Palabras clave: Modelo praxeológico- Inclusión- Inteligencias múltiples-Educación para la paz-Liderazgo.

Keywords: Praxeology model - Inclusion - Intelligence type - Peaces courses - Leadership. 


\section{INTRODUCCIÓN}

La investigación surge como resultado de cuestionamientos frente a la extenuante lucha colombiana y el papel de los jóvenes frente al establecimiento de un país con mayores oportunidades desde los espacios educativos, caracterizados por ser los principales ejes de transformación y cohesión del tejido social. Espacios en los cuales, los jóvenes tienen la posibilidad de empoderarse, interpretar, proponer y generar mecanismos educativos incluyentes hacia la construcción de paz.

El conflicto colombiano es competencia de todos; representa una problemática con fuerte incidencia en la sociedad, pues como se menciona en la ponencia de Caroline Moser titulada «El costo social del conflicto e iniciativas locales de paz en Colombia», se permite diferenciar la evolución de la incidencia del conflicto en la sociedad; que inicialmente afectaba solo a los actores directamente involucrados, y que posteriormente resultó afectando a toda la población del país (Fundación Ideas para la Paz, 2004). Es por esto, que conjuntamente debe lucharse por la paz; para de este modo, darle un fin. Posteriormente, entrar a trabajar en temas de postconflicto y todos los retos que esto implica, que en manos de jóvenes con liderazgo y empoderamiento lograrán brindar y construir un mejor futuro para las nuevas generaciones.

Cabe mencionar que la población colombiana ha evidenciado 60 años de conflicto, período caracterizado por crímenes de lesa humanidad, violencia sexual, desplazamiento forzado, secuestro, reclutamiento de menores, torturas, entre otros; que han marcado su historia y la de cada uno de sus habitantes. Sin embargo, extenuantes luchas por un período de transición se han llevado a cabo, con cerca de seis procesos de paz fallidos y uno que a la fecha se encuentra en proceso de desarrollo.

A razón de lo mencionado anteriormente y teniendo en cuenta investigaciones previas realizadas en la Corporación Universitaria Minuto de Dios sede Zipaquirá surge el planteamiento final ¿de qué manera el modelo praxeológico promueve la formación de liderazgo en los estudiantes del Semillero Adherencia Cognitiva y Cambio Social del programa de Trabajo Social para instaurar sesiones de cátedras de paz desde la potencialización de sus inteligencias múltiples? Tomando como base la necesidad de capacitar a los jóvenes desde este modelo propuesto por la institución; mediante el cual es posible crear sesiones de cátedras de paz con una acción pedagógica innovadora desde el empoderamiento de los jóvenes del Semillero para trabajar conjuntamente por la paz.

Pascual (2006) expone en su libro «clarificación de valores y desarrollo humano»; la situación actual de injusticia social y la misma naturaleza del hombre, temporal e histórica, exigen que la determinación de los valores-fines de la educación sea hecha en una perspectiva dinámica, de modo que el proceso educativo atienda consciente y directamente a la formación de actitudes para el cambio. Por lo que se refiere a la realidad y su dinamismo, como ejes fundamentales para empezar a trabajar con premura en los diferentes aspectos que tienen una relación directa con los procesos educativos y su articulación con valores y competencias ciudadanas que permitan un mayor desarrollo y apropiación de lo que sucede en el entorno; a través de relaciones interpersonales, (según Raths citado por Pascual, 2006, p: 36) cada persona tiene que adquirir con esfuerzos y luchas su propio conjunto de valores si desea llegar una existencia satisfactoria. Lo que les interesa es el proceso por el cual llegamos a asumir como propios los valores, de tal modo que esos valores lleguen a influir realmente y sean, por lo tanto, guías de nuestra conducta. Es por esto que anteriormente se realizaron en la Universidad investigaciones que permitieran determinar e identificar la manera en la cual los estudiantes reconocían estos procesos dentro de su quehacer como futuros profesionales; así como también evaluar el nivel de conocimiento frente a los sucesos históricos que marcaron la historia del país.

De acuerdo con las variables de estudio fue posible determinar la importancia del empoderamiento y el liderazgo, así como también el trabajo en valores desde el aula, como principales promotores de procesos de transformación y reconocimiento del contexto; con base en una relación o contacto directo con la realidad.

Uniminuto (s.f), define la praxeología como un discurso (logos), construido después de una seria reflexión, sobre una práctica particular y significante (praxis), como un procedimiento de objetivación de la acción, como una teoría de la acción; por el tipo de análisis que realiza pretende hacer que dicha praxis sea más consciente de su lenguaje, de su funcionamiento y de lo que en ella está en juego, sobre todo del proceso social en el cual el actor o practicante está implicado y del proyecto de intervención que construye para cualificar dicho proceso; todo esto con el fin de acrecentar su pertinencia y su eficacia liberadora. Ella es el resultado, entonces, de un análisis empírico y de un 
discurso crítico: la praxeología designa, desde el principio, una reflexión práctica sobre los principios de la acción humana y de sus técnicas, pero busca, igualmente, los principios generales y la metodología adecuada para una acción eficaz y pertinente. De este modo, las investigaciones permitieron inferir la trascendencia del modelo praxeológico de la Corporación Universitaria Minuto de Dios como un instrumento que genera en los estudiantes las bases para un pleno desarrollo personal y profesional, que puede ser implementado en la articulación de nuevos mecanismos y herramientas pedagógicas que se trabajen desde el día a día en el aula, teniendo en cuenta las habilidades y potencialidades de cada estudiante.

Tal como se mencionó anteriormente, desde las instituciones educativas se hace posible contribuir y proponer acciones pedagógicas innovadoras que se trabajen desde y para la comunidad en pro de un empoderamiento, que poco a poco se oriente hacia la cohesión y repare el tejido social, cuyo fin radica en procurar la construcción de paz.

Es por esto, que la formación de líderes hoy día constituye y representa un factor trascendental en los diferentes procesos que se desarrollan en la sociedad desde lo local hasta lo global. Colombia, no es la excepción y más cuando se encuentra en este importante proceso de transición hacia un estado de paz. Es por esto, que se hace necesaria la implementación y capacitación desde la Corporación Universitaria Minuto de Dios, para lograr en los jóvenes un liderazgo orientado a temas de paz y conocimientos históricos, y con ello poder llegar a establecer sesiones de cátedras de paz que permitan un empoderamiento y transformación hacia una sociedad más inclusiva, con cultura cívica, tolerante y con una paz estable y duradera.

\section{MODELO PRAXELÓGICO}

La Corporación Universitaria Minuto de Dios - UNIMINUTO se compromete con una educación para el desarrollo humano y social integral, a través de la formación de líderes e innovadores sociales, constituidos como seres humanos íntegros, y profesionales éticos y competentes, para construir un país justo, reconciliado, fraternal y en paz. Para ello ha adoptado un enfoque pedagógico praxeológico que integra el saber (teoría) y la práctica (praxis) mediante un proceso reflexivo que parte del análisis crítico de las prácticas y experiencias de cada persona o comunidad, llevándolas a integrar su(s) proyecto(s) de vida personal y profesional, a un proyecto de transformación de la sociedad.

El modelo praxeológico de la Corporación Universitaria Minuto de Dios promueve el desarrollo de todas las dimensiones de la persona, partiendo de las potencialidades de cada uno y tiene en cuenta el conjunto de ideas, creencias, valores, actos, palabras y contextos del estudiante o comunidad, para ayudarle(s) a construir su bienestar personal y comunitario. (Juliao, 2012).

El modelo praxeológico de la corporación Universitaria Minuto de Dios es un referente significativo puesto que es necesario en la formación de personas íntegras y críticas, para que con este modelo se incentive y promueve la participación de los estudiantes hacia la implementación de cátedras de paz a través de líderes estudiantiles.

\subsection{Dimensiones de la persona}

El ser humano, como todo ser vivo, no es un agregado de elementos yuxtapuestos; es un todo integrado que constituye un supra sistema dinámico, formado por muchos subsistemas perfectamente coordinados: el subsistema físico, el químico, el biológico, el psicológico, el social, el cultural, el ético-moral y el espiritual (Martínez, 2009). Es mediante la integración de todos éstos que se constituye la personalidad; razón por la cual, UNIMINUTO juega un papel trascendental con la implementación de su enfoque praxeológico, que incide notablemente en la potencialización de la dimensión humana de sus estudiantes.

El desarrollo de las áreas del ser humano desde las primeras etapas de vida es muy importante, puesto que esto influencia su proceso y crecimiento en la sociedad, dando inicio en la familia que es la principal base donde se recibe afecto y se empieza a formar la conducta, los lazos afectivos y sociales del menor; estableciéndose por medio de las pautas de crianza, estilos de vida, creencias religiosas, principios y valores. 
Los centros educativos también ocupan un papel importante en el desarrollo de las dimensiones del ser humano, porque desde allí, es donde se empieza a dar en mayor escala la potencialización de estas dimensiones, que le permitan desarrollar sus cualidades de líder, para encaminar y transformar diferentes procesos sociales como la implementación de una cultura y cátedras de paz.

Al analizar cada una de estas dimensiones se puede observar como los factores internos y externos a la persona pueden afectar su desarrollo integral, condicionando poco a poco sin que este pueda darse cuenta. En este proceso se hace necesario tener unas condiciones de vida óptimas, suplir las necesidades, apoyar los procesos pedagógicos, y sociales que generen impacto y apego y propicien un ambiente sano y confiable, donde se siente feliz y libre para ser como es y dar todo lo mejor de sí.

\subsection{Componentes y fases}

De acuerdo con Juliao (2012), «se da inicio con la fase ver, desde una mirada pedagógica, la cual involucra un espacio de exploración, análisis o síntesis; seguido del elemento didáctico sustentado en el proceso empírico o experimental, que conlleva al tercer elemento epistemológico que refiere a la adquisición de conocimientos. Posteriormente, se encuentra la fase juzgar, en la cual los procesos pedagógicos surgen desde problematizar la observación en conjunto con la formulación de hipótesis; continuo de la estructuración de los discursos en el elemento didáctico, que dan paso a la revisión de las fuentes teóricas desde la parte epistemológica. En la tercera fase, que corresponde al actuar se evidencia la comprensión de los procesos partiendo de la identificación de problemáticas y determinación de las finalidades desde la visión pedagógica, por otro lado, el elemento didáctico abarca los campos de práctica y modos de acción. Por lo cual, se realiza la construcción de proyectos, la gestión de las acciones y la evaluación de los mismos desde el componente epistemológico. Por último, se abarca la fase de devolución creativa que permite una evaluación prospectiva desde cada uno de los elementos: a nivel pedagógico permitiendo la formulación de ¿qué aprendemos de lo que hacemos?, un actuar y nuevas vías de acción, un cambio a partir del fundamento didáctico y una intervención a mediano y largo plazo a través de la epistemología».

\section{LIDERAZGO}

Decir «líderes» es pensar en aquellos hombres y aquellas mujeres que cambiaron el curso de sus sociedades, conduciéndolas a un futuro distinto del que -suponemos- habrían tenido de no mediar su existencia (Rojas \& Gaspar, 2006).

El liderazgo resulta trascendental en el momento de actuar sobre un determinado grupo de personas, además, el líder ejerce una influencia conduciendolos hacia una línea de pensamiento. Por consiguiente, el liderazgo en el proceso de educación que refleja ser importante porque requiere de compromiso por parte del cuerpo administrativo y docente, en el cual deben identificarse con los sucesos violentos de conflicto armado y miles de afectados que ha dejado la violencia por más de 60 años en el país, donde los altos grados de indiferencia pueden llevar a la confrontación del ideal de una paz estable y duradera. Desde este proceso se puede exponer una visión amplia acerca del posconflicto en Colombia, partiendo de la formación de líderes y la transformación, la cual se logra desde la percepción de cada uno de los docentes (transmisores de conocimiento) y de qué manera ellos aplican nuevos modelos de enseñanza que favorezcan el liderazgo estudiantil puesto que los aprendizajes escolares ocurren en el aula de clase.

Según Rojas \& Gaspar (2006) se introduce el tema del liderazgo en educación a partir de las bases éticas, políticas y metodológicas que son parte de la visión de la OREALC/UNESCO. Para introducir a estos aspectos fundamentales, nos referimos a los líderes como personas o grupos de personas competentes en el arte de conducir a una comunidad en la construcción de un futuro deseable por o para su comunidad. Esto se aprecia claramente en algunos personajes en la historia del siglo XX, como Mahatma Ghandi o Winston Churchill, quienes supieron observar las frustraciones y deseos de sus comunidades y encabezaron procesos sustentados en la promesa de un mejor porvenir. El segundo capítulo se refiere a personalidades de diferentes países y ámbitos que fueron líderes de sus naciones a lo largo del siglo XX y que nos ayudan a ejemplificar las características que los educadores y directivos latinoamericanos deben desarrollar para mejorar la calidad de la educación en sus escuelas. El tercer capítulo de la obra define el origen y las causas de los aprendizajes escolares. En 
ese sentido, se parte de la premisa según la cual los aprendizajes que ocurren en las salas de clases son responsabilidad principal de los profesores y profesoras. El liderazgo ayuda a los directivos y miembros de una comunidad educativa a crear una mirada propia sobre el aprendizaje en la escuela a partir de una gestión impecable y fundada en alianzas significativas. En el capítulo cuarto se aborda un rasgo fundamental en la tarea de las escuelas de calidad: la construcción de climas escolares efectivos. Para este fin, el lenguaje y las emociones son dos elementos que están estrechamente vinculados. el último capítulo define la motivación que debe generar el directivo en su escuela. La motivación es un estado de ánimo que debe existir en todos los miembros de la escuela, por consiguiente, un director o directora líder sabe generar climas adecuados para la Introducción - XV adquisición de aprendizajes en condiciones difíciles.

Existen varias características para reconocer un líder positivo y negativo según Maier (s.f) un líder positivo es aquel que piensa en positivo y cultiva un clima positivo, establece una comunicación asertiva, fluyen emociones agradables en nuestro cerebro, produciéndose más dopamina y serotonina, que son neuroquímicos relacionados con el placer.

El líder negativo siempre estará humillando y rebajando a sus subalternos, pues sólo se sentirán líderes si los demás se muestran inferiores a ellos, les temen y les obedecen. Desmotiva, aísla, desintegra, producen sentimientos que afectan la estabilidad emocional, al poco tiempo. Prefiere a gente sin carácter, que haga todo lo que él dice sin oponerse, que aprovechan su capacidad e inteligencia para liderar, pero hacia aspectos negativos.

Por medio de las cátedras de paz se orientará a los líderes positivos a que no se dejen afectar negativamente, y en cambio enseñar y promover un liderazgo positivo para la construcción de una sociedad.

\subsection{Limitaciones}

El desarrollo adolescente se da en una delicada interacción con los entes sociales del entorno; tiene como referente no sólo la biografía individual, sino también la historia y el presente de su sociedad. Es el periodo en que se produce con mayor intensidad la interacción entre las tendencias individuales, las adquisiciones psicosociales, las metas socialmente disponibles, las fortalezas y desventajas del entorno (Krauskopf, 1994,1995).

Lutte (1991) plantea que, actualmente, se va haciendo difícil distinguir entre adolescencia y juventud y, por ello, los autores más destacados en la materia y diversas instituciones no coinciden en su diferenciación, fases ni en los procesos que sistematizan. Se maneja una imagen fragmentada de la vida en la fase juvenil que puede orientarse en diversas direcciones; incluso se superponen los conceptos de adolescencia y juventud cuando se fijan los límites de la primera entre los 10 y los 20 años y los límites de la segunda entre los 15 y los 25 . Aun cuando en sus extremos son poblaciones que se encuentran en estadios diferentes desde el punto de vista psicosocial y económico, en el diseño de las políticas y sus paradigmas, existen aspectos relacionados que afectan a ambos grupos cronológicos.

Actualmente nos encontramos en un periodo de transición donde se busca la igualdad para todos los seres humanos sin importar la condición social, sexual, religiosa, política o cultural, pero esto no ha sido muy fácil debido a las diferentes concepciones y paradigmas que se han creado frente a los diversos procesos de cambio que se plantean para evitar que haya una exclusión o segregación ante los nuevos procesos.

Se evidencia que hay adultos que aún se resisten a la idea de que los jóvenes puedan a su corta edad a realizar procesos que no vayan de acuerdo a lo planteado por ellos, aunque se sientan con el deber moral de ser ellos los pioneros y no los jóvenes que a su concepto no tienen la experiencia que ellos han forjado a través de los años. También se observa esa lucha de poderes entre lo ya establecido y los nuevas formas de conocimiento que dan inicio a ideas innovadoras que pueden trascender en el tiempo, pero a la vez esta lucha puede hacer que los jóvenes se sientan vulnerados y decidan excluir o dejar de lado la realidad colectiva, perdiendo a si el interés en lo que les conviene para su vida y dejando esa labor a criterio de los adultos, que pueden no tener totalmente la razón por estar arraigados en métodos y pensamientos que no se ajustan a la situación y deben ser reestructurados para que así, puedan dar resultados que beneficien a todos. 
Se deben establecer espacios donde los jóvenes y los adultos dialoguen, desde el punto de vista de las dos partes se llegue a un acuerdo donde las ideas de las persona adultas con las de los jóvenes puedan dar vía a nuevas formas de participación y a un trabajo mancomunado que permita el avance y no la división de estas partes.

Los jóvenes perciben las cosas de otra manera debido al procesos de vida que se dan actualmente, con una serie de conocimientos y experiencias, que los llevan a tener otra visión del mundo, lo cual hace necesario que se visibilicen en la sociedad para así empoderarse de los diferentes proyectos enfocados en la Juventud y participen de manera constante en la formación e implementación de los nuevos conocimientos, formando así una generación de jóvenes líderes apoyados en los conocimientos de los adultos para trascender las barrera multiculturales.

\section{INTELIGENCIAS MÚLTIPLES}

El ser humano se encuentra en un constante proceso de evolución y aprendizaje, desde su nacimiento está condicionado a espacios que requieren su comprensión del entorno y conocer cada una de las cosas que lo rodean. Para esto, hace uso de la inteligencia, como factor innato del individuo, que se define como la capacidad cerebral por la cual conseguimos penetrar en la comprensión de las cosas eligiendo el mejor camino. La formación de ideas, el juicio y el razonamiento frecuentemente señalados como actos esenciales de la inteligencia, como facultad de comprender (Antunes, 2006, p.9).

Igualmente, se relacionan inteligencias múltiples en cada individuo teniendo en cuenta recientes investigaciones en neurología que sugieren la presencia de zonas en el cerebro humano (...) zonas que según Gardner serían ocho y por lo tanto, el ser humano poseería ocho zonas de su cerebro dónde se albergarían diferentes inteligencias, lo que él denomina inteligencias múltiples y son lingüística o verbal, lógico- matemática, la espacial, la musical, la cinestésica corporal, la naturista y las inteligencias personales (Antunes, 2006, p.20).

De este modo, se infiere que cada uno de los estudiantes del semillero adhiere y representa su percepción de las problemáticas y realidades de acuerdo a su capacidad cerebral dada por el estímulo y desarrollo de cualquiera de las inteligencias.

\section{CÁTEDRAS DE PAZ}

En épocas de posconflicto es normal escuchar hablar de temas orientados a la paz, su incidencia en la sociedad y miles de cuestionamientos frente a la manera más adecuada de fomentarla y mantenerla. Una de las principales respuestas, radica en la educación como principal herramienta y mecanismo de transmisión, potencialización y aplicación de conocimientos que permiten transformar la realidad social, por esto, se habla de educación para la paz.

\subsection{Educación para la paz}

La educación para la paz consiste en fomentar la capacidad de apreciar la libertad y las aptitudes que permiten responder a sus retos a partir de la preparación de sus ciudadanos para que sepan enfrentarse y afrontar las diversas situaciones que deben presenciar (UNESCO 1995 citado por Labrador).

Es así como en palabras de Johan Galtung: educar para la paz es enseñar a la gente a encararse de manera más creativa, menos violenta, a las situaciones de conflicto y darle los medios para hacerlo (Labrador, 2000), es así como debe hablarse de una educación para la paz que esté apoyada en valores fomentados y trabajados desde los jóvenes que se empoderan y autogestionan los intereses de su comunidad universitaria y de su entorno, para una mejor calidad de vida caracterizada por una cultura de paz inclusiva.

Reflexionar sobre lo que podría ser la educación para la paz es una verdadera necesidad y un reto de primera magnitud (Labrador, 2000), razón por la cual, para la Universidad Minuto de Dios es imperante y primordial empezar a pensar en educación para la paz, y más teniendo en cuenta el período histórico 
por el cual está pasando el país. Se hace necesario que los jóvenes tengan pleno conocimiento de los diferentes sucesos que marcaron al país y puedan empezar a pensar en mecanismos de no repetición, en herramientas pedagógicas que puedan instaurarse y permitan la transmisión de conocimientos íntegros y críticos, que sean la pauta para las diferentes transformaciones sociales que abarca e implica vivir en una cultura de paz.

Según Herrero (2003) si se quiere que una educación para la paz sea posible en el sistema educativo, además de examinar las cuestiones didácticas y organizativas, se debe dedicar una atención especial a las cuestiones sociales, políticas y filosóficas que intervienen en el currículum. Es necesario replantear el currículum actual del sistema educativo para superar sus muchas violencias institucionalizadas. En síntesis, se podría terminar diciendo que otro de los pilares básicos de la educación para la paz que propone Jares es que el proceso enseñanza-aprendizaje esté basado en el enfoque socio-afectivo, la inclusión de los aspectos afectivos y sentimentales a la educación, además de los intelectuales. Cabe destacar que para el autor la perspectiva práctica de la educación para la paz es su reto principal. Aplica, por tanto, los presupuestos y conceptos de la educación para la paz a ejemplos prácticos como juegos y ejercicios de simulación, dinámicas de clarificación de valores, juegos de roles, estudios de casos, dilemas morales, análisis de textos literarios.

Se puede analizar y reafirmar como la educación y cultura para la paz en el aula se debe iniciar con la creación de una comunidad basada en la comunicación y donde todas las relaciones interpersonales se deben dar en reciprocidad, confianza, comprensión, empatía y seguridad. También conocer las tres características del método socioafectivo con las estrategias que puede realizar el participante para encontrar las falencias por las cuales no se ha podido realizar la transición de la cultura de violencia a la cultura de paz que tanto se desea. De igual manera adoptar algunas metodologías estratégicas que ayudan a cooperar para junto al crecimiento del liderazgo positivo, se logre perdurar con una cultura de paz estable y equitativa, sin dejar de lado el contexto social en el cual se desarrolla cada ser humano, lo cual definitivamente es un reto.

\subsection{Trabajar para una cultura de paz}

Cultura de paz es una cultura fundamentada en valores que inspiran comportamientos y actitudes ciudadanas propicios para la convivencia armónica y pacífica. Construir una cultura de paz es arraigar en la conciencia de las personas y de las instituciones el convencimiento de que la vigencia, vivencia y práctica de relaciones sociales y humanas fundamentadas sobre el respeto de los derechos humanos, es un imperativo categórico para la viabilidad de una sociedad (Alianza Educación para la Construcción de Cultura de Paz, 2005).

Es posible construir y trabajar para una cultura de paz desde la comunidad (Ángulo, 2005, p. 13), en este caso: el semillero y las mujeres desplazadas, desde tres tipos de aprendizaje; como lo son: aprendizaje de la interdependencia, el pluralismo y por último la solidaridad. Mecanismos, que, al ser implementados, pueden generar grandes transformaciones desde la comunidad estudiantil, para la sociedad.

\section{DISCUSIÓN Y CONCLUSIONES}

- Con el proceso investigativo ha sido posible reconocer la importancia de la aplicación del modelo praxeológico, en prácticas que involucran el liderazgo estudiantil encaminado a la instauración de cátedras sustentadas en una educación para la paz, en la cual se reconozcan los diferentes sucesos históricos, el papel del individuo en sociedad y su incidencia en el entorno social del cual forman parte, a partir de la apropiación de las competencias ciudadanas.

- El modelo praxeológico de la Corporación Universitaria Minuto de Dios sede Zipaquirá, basado en ver, juzgar, actuar y devolución creativa, es fundamental para la cooperación, fortalecimiento de capacidades y habilidades de los estudiantes.

- El liderazgo en los estudiantes es trascendental en tanto permite la articulación de procesos inclusivos enfocados a la construcción de espacios de adherencia al conocimiento y transmisión de saberes. 
- Con el reconocimiento y potencialización de las inteligencias múltiples es factible establecer herramientas pedagógicas innovadoras sustentadas con actividades lúdico didácticas enfocadas a la construcción de paz.

- Cada estudiante del semillero está en la capacidad de reconocer sus fortalezas y debilidades, trabajar desde ellas para la promoción de ideas que transformen la realidad social.

- Debe hablarse de una educación para la paz que esté apoyada en valores fomentados y trabajados desde los jóvenes que se empoderan y autogestionan los intereses de su comunidad universitaria y de su entorno, para una mejor calidad de vida caracterizada por una cultura de paz inclusiva.

\section{REFERENCIAS BIBLIOGRÁFICAS}

Antunes, A. (2006). Inteligencias múltiples: cómo estimularlas y desarrollarlas. Madrid, España. El comercio S.A.

Gardner, H. (s.f). Inteligencias múltiples la teoría en la práctica. Barcelona, España. PAIDÓS. Versión en línea http://brd.unid. edu.mx/recursos/CIED/Gardner_inteligencias.pdf.

Herrero, S. (2003). Educar para la paz. Un reto en el nuevo milenio. Convergencia 33: Revista de ciencias sociales septiembre-diciembre 2003. Toluca México. Versión en línea http://www.redalyc.org/articulo.oa?id.

Juliao, C. (2012). Enfoque praxeológico UNIMINUTO. Archivo de video, recuperado de https://www.youtube.com/watch?$\mathrm{v}=\mathrm{i} 048 \mathrm{oJCFNeA \&} \mathrm{t}=121 \mathrm{~s}$.

Labrador, C. (2000). Educación para la Paz y la Cultura de Paz en documentos Internacionales. Contextos Educativos file:///C:/ Users/Toshiba/Downloads/Dialnet-EducacionParaLaPazYCulturaDePazEnDocumentosInterna-201070\%20(4).pdf.

Maier, V (s.f.). Universidad de Aconcagua. Versión en línea https://alumnosenfermeriauac2014.files.wordpress.com/2014/0/ sesion_u_1_7_liderazgo_positivo_y_negativo.pdf.

Palos, J. (s.f.). Educación y cultura de la paz. Universidad de Barcelona. Organización de Estados Iberoamericanos: Para la Educación la Ciencia y la Cultura. Recuperado de http://www.oei.es/historico/valores2/palos1.htm.

Pascual, A. (2006). Clarificación de valores y desarrollo humano: estrategias para la escuela. Madrid, España. MARCEA, S.A. 